\title{
Pharmacophore Modeling and Docking Study of Pyrazolylaminoquinazoline Derivatives as Highly Potent Fibroblast Growth Factor Receptor Inhibitors2 (FGFR2)
}

\begin{abstract}
LUMINITA CRISAN, DANIELA VARGA, LILIANA PACUREANU*
Institute of Chemistry Timisoara of Romanian Academy, Department of Computational Chemistry, 24 Mihai Viteazul Blvd., 300223, Timisoara, Romania

In this study pharmacophore modeling and molecular docking investigations have been performed on pyrazolylaminoquinazoline derivatives, highly potent fibroblast growth factor receptor2 (FGFR2) inhibitors. The best pharmacophore hypotheses displaying five features (ADHRR.2051 and AADHR. 798) were generated using a set of 28 compounds. The associated $3 \mathrm{D}$ atom-based quantitative structure - activity relationships $(Q S A R)$ models were statistically robust showing high correlation coefficients $(R$-squared $=0.981$ / 0.982$)$, and cross validation coefficients ( $Q$-squared $=0.645 / 0.671)$. The $R$-Pearson values for the test set of 0.805 / 0.820 indicate that the models are robust and exhibit good predictive power. The interactions of pyrazolylaminoquinazoline with FGFR2 binding site revealed two hydrogen bonds with Ala567. The obtained pharmacophore, 3D atom-based QSAR models and binding features resulted from docking studies can help medicinal chemists to design new pyrazolylaminoquinazoline inhibitors with improved potency.
\end{abstract}

Keywords: Pharmacophore modeling, QSAR, docking, pyrazolylaminoquinazoline

Fibroblast growth factor receptors (FGFRs) are included into receptor tyrosine kinase (RTK) family [1] and are currently targeted for novel oncologic therapies. FGFRs are involved in several critical biological processes including morphogenesis, differentiation, patterning, and cell migration [2]. They network with multiple fibroblast growth factors (FGFs), to stimulate development [2]. The FGFR2 gene is confined on the chromosome 10q26.13 displaying about 21 exons which by alternative splicing spawn multiple protein isoforms [3]. FGFR isoforms include seven receptors, as follows: FGFR1b, FGFR1c, FGFR2b, FGFR2c, FGFR3b, FGFR3c and FGFR4 [4]. The kinase domain of FGFR2 is situated in the area delimited by the residues 458-768.

Today's computational chemistry is widely involved in early stages of drug discovery programs. Ligand- and structure-based virtual screening is efficient approaches which can reduce large chemical libraries of compounds to a smaller list of potentially active molecules [5, 6]. Pharmacophore modeling is one of the successful tool in computer-aided drug discovery and development which can be applied in various stages i.e., virtual screening, lead optimization, de novo design, side effect / off-target prediction and target identification. Pharmacophore modeling identifies the key elements of molecular recognition and help to correlate the observed biological activities of chemical compounds with their chemical structures. Pharmacophore models are useful tools to mine chemical databases to identify new chemical entities with various scaffolds (the so called scaffold-hopping potential) [6-9]. In drug design quantitative structure-activity relationships (QSAR) is a concept which can contribute markedly to accurate prediction of biological activities. QSAR rely on the hypothesis that fluctuation of the biological activity of the compounds is associated with differences in their structural characteristics [7]. Besides biological activity prediction, another aim of QSAR analysis is to direct ligand-based design of new analogue series [8, 10]. Molecular docking allows to explore the interaction pattern of a small molecule with a biological target and to confirm the conclusions of pharmacophore and QSAR investigations [11-13]. Current interest in drug design is related to pharmacophore models which are linked to molecular docking to consensually improve virtual screening results [11, 12].

To the best of our knowledge, the series of 28 pyrazolylaminoquinazoline derivatives [14] - highly potent FGFR2 inhibitors have not been investigated computationally [14]. Therefore, the current work aims at using relevant structural information from these ligands to develop pharmacophore models. The pharmacophore models will be validated by 3D atom-based QSAR and docking to provide an extensive picture of pyrazolylaminoquinazoline interaction with FGFR2.

\section{Experimental part \\ Dataset}

A dataset comprising 28 pyrazolylaminoquinazoline derivatives potent fibroblast growth factor receptor inhibitors, FGFR2 (table 1) were collected from a recent publication [14] and used in the current study. The molecular structures of all 28 pyrazolylaminoquinazoline derivatives were sketched with the help of Symyx Draw software [15] and saved as smiles codes.

\section{Pharmacophore 3D-QSAR modeling}

Phase module [16-18] of Schrodinger 2016-1 suite [19] was used to develop the pharmacophore hypotheses and their corresponding 3D-QSAR models for 28 pyrazolylaminoquinazoline derivatives. The structures were prepared using the standard protocol including generation of ionization states and tautomers at physiological $\mathrm{pH}$ $(7.4 \pm 0.2)$, remove salts [20], conformational expansion and energy minimization based on the OPLS-2005 force field [21, 22]. The experimental biological activities, $I C_{50}$ were transformed into negative logarithm of inhibitory concentrations, $\mathrm{plC}_{5^{\circ}}$. In the pharmacophore generation step the threshold for active compounds was set to $\mathrm{plC}_{50}$ $>9.3$ (8 compounds in picomolar range), for inactives was 
Table 1

THE STRUCTURES OF PYRAZOLYLAMINOQUINAZOLINE DERIVATIVES, THEIR EXPERIMENTAL (PIC50 ${ }_{\text {EXP }}$ ) AND PREDICTED (PIC50 PRED ) ACTIVITY VALUES OBTAINED BY 3D ATOM BASED QSAR

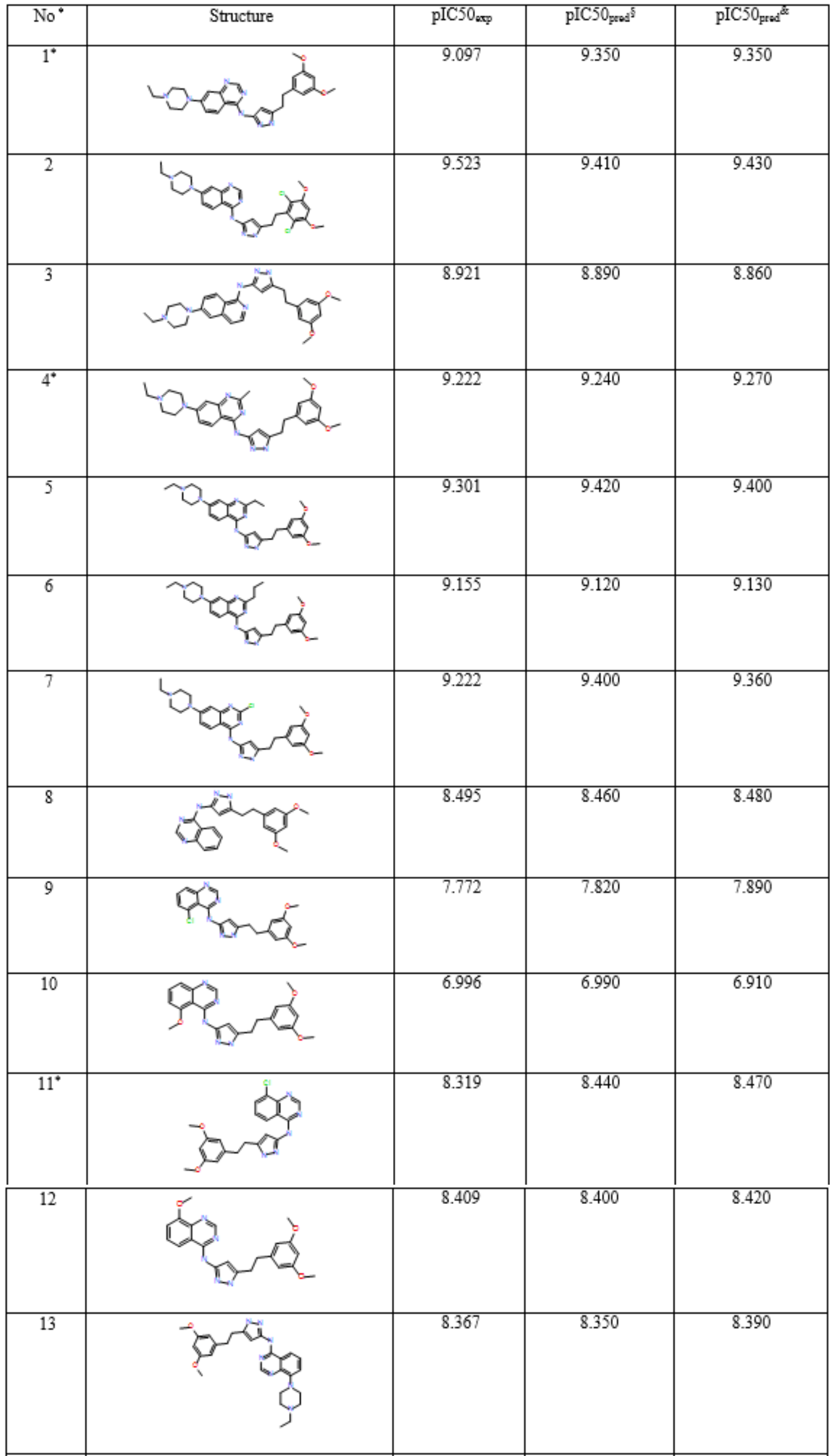


Table 1 - CONTINUATED

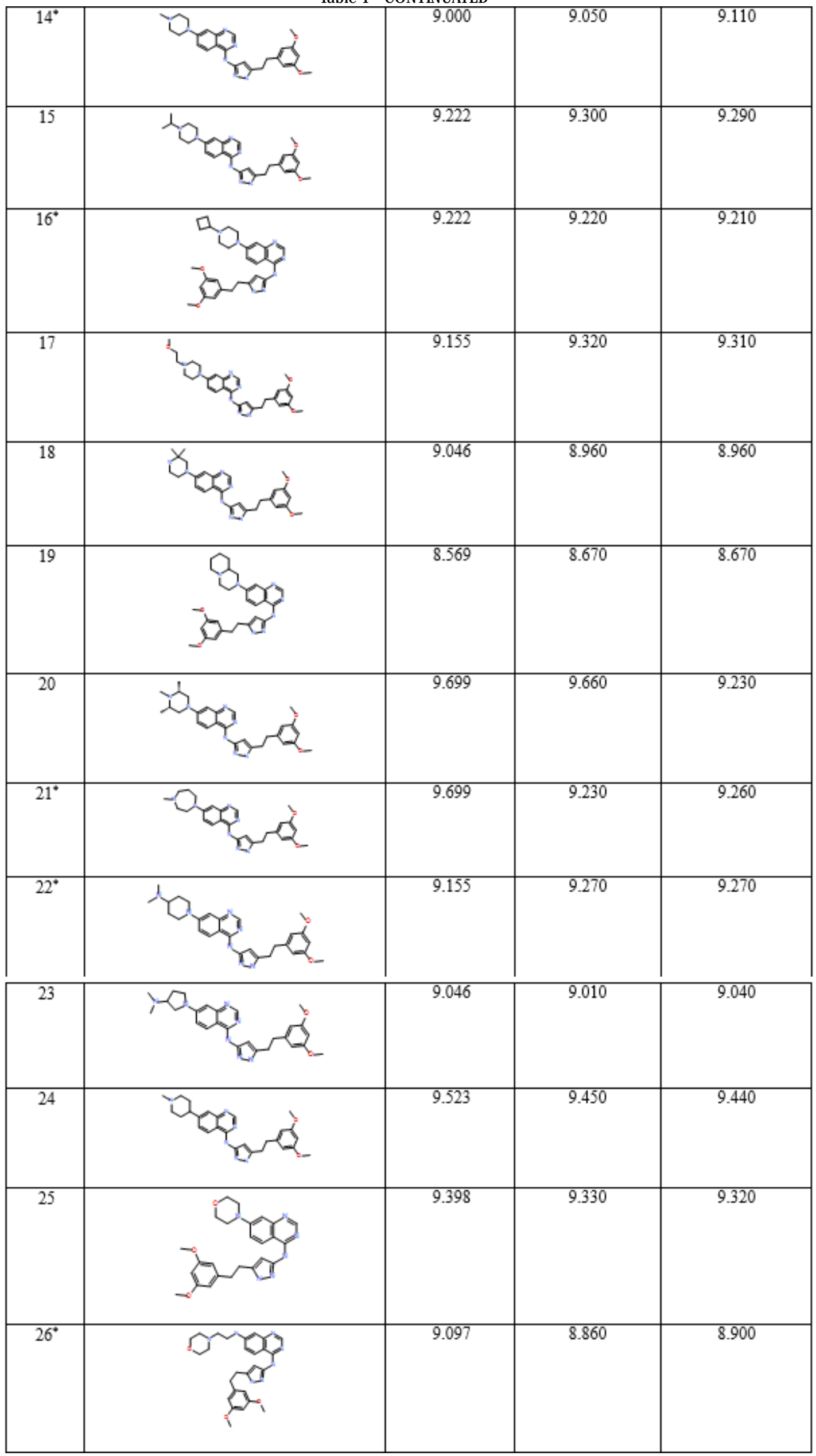




\begin{tabular}{|l|c|c|c|c|}
\hline 27 & 9.398 & 9.250 & 9.260 \\
\hline $28^{*}$ & & & & \\
Table 1 - CONTINUATED \\
\hline
\end{tabular}

$\mathrm{plC}_{50}<8.5$ (6 compounds) while the rest of the inhibitors were considered as moderately active. Redundant conformations were removed if heavy atoms root mean squared deviation (RMSD) is greater than $2 \AA$. Phase recognizes the following built-in pharmacophore features: acceptor (A), donor (D), hydrophobic (H) and ring $R(R)$. In the first step Phase detect the shared set of pharmacophore features relying consistently in actives which display common spatial arrangements. The ranking of pharmacophore hypotheses in order to select the best alignment of the active compounds was carried out using a threshold RMSD of $1.2 \AA$.

3D atom-based QSAR, consider a molecule as consisting of overlapping van der Waals spheres displaying the following properties: hydrogen-bond donor (D), electron withdrawing (i.e. including hydrogen bond acceptors, W), hydrophobic $(H)$, negative ionic $(N)$, positive ionic $(P)$, and miscellaneous $(X)$ [18]. In order to build the 3D atom-based QSAR model, the data set was randomly split into training set $(67 \%)$ and test set (33\%) used for validation purposes. The atom-based 3D-QSAR model have been developed at three PLS factors in order to avoid overfitting. Automatically, Phase build atom-based 3D QSAR models for all pharmacophore hypotheses developed. Internal and external validation parameters for atom-based 3D QSAR were: squared correlation coefficient R-squared (training set), squared correlation coefficient Q-squared (test set), standard deviation (SD), Pearson's correlation coefficient R-Pearson (test set), RMSE - the root-mean-square error in the test set predictions, statistical significance $(P)$, and Fisher test (F) [20].

\section{Molecular docking}

All ligands were prepared for docking by generation of ionization states and tautomers with LigPrep software, followed by conformational expansion using OMEGA module [23-27] from OpenEye package, retaining a maximum of 200 conformers per compound, an energy cut-off of $10 \mathrm{kcal} / \mathrm{mol}$ and RMSD less than $0.8 \AA$. The 3D structure of FGFR2 kinase domain was extracted from the co-crystal 1OEC deposited in PDB [28] and prepared for docking with Fred Receptor [29] module version 2.2.5 from OpenEye package. The active site box was set to $6933 \AA^{3}$, inner contour of $534 \AA^{3}$ and outer contour of $2341 \AA^{3}$, whereas crystallographic water molecules were deleted. The docking procedure was carried out using Fast Rigid Exhaustive Docking (FRED) [30-32] version 3.0, to perform a rigid receptor-flexible ligand docking of pyrazolylaminoquinazoline derivatives.

\section{Results and discussions \\ Pharmacophore modeling}

On the basis of performance various pharmacophore models generated were ranked as function of significant statistical parameters R-squared, SD, F, P, RMSE, Q-squared, and Pearson-R. According to the above criteria the best pharmacophore models were selected. A number of ninetytwo pharmacophore hypotheses from the total one thousand-six variant hypotheses generated have an RPearson $>0.8$ and Q-squared $>0.6$. Two pharmacophore hypotheses were selected based on statistical parameter values and survival-inactives score. The best QSAR models resulted are based on the alignments provided by pharmacophore hypotheses ADHRR.2051 and AADHR.798: R-squared of 0.981 and 0.982 , Q-squared values of 0.645 and 0.641 , RMSE values of 0.204 and 0.196 , R-Pearson of 0.805 and 0.820 , indicating that the models are robust and have good predictive power.

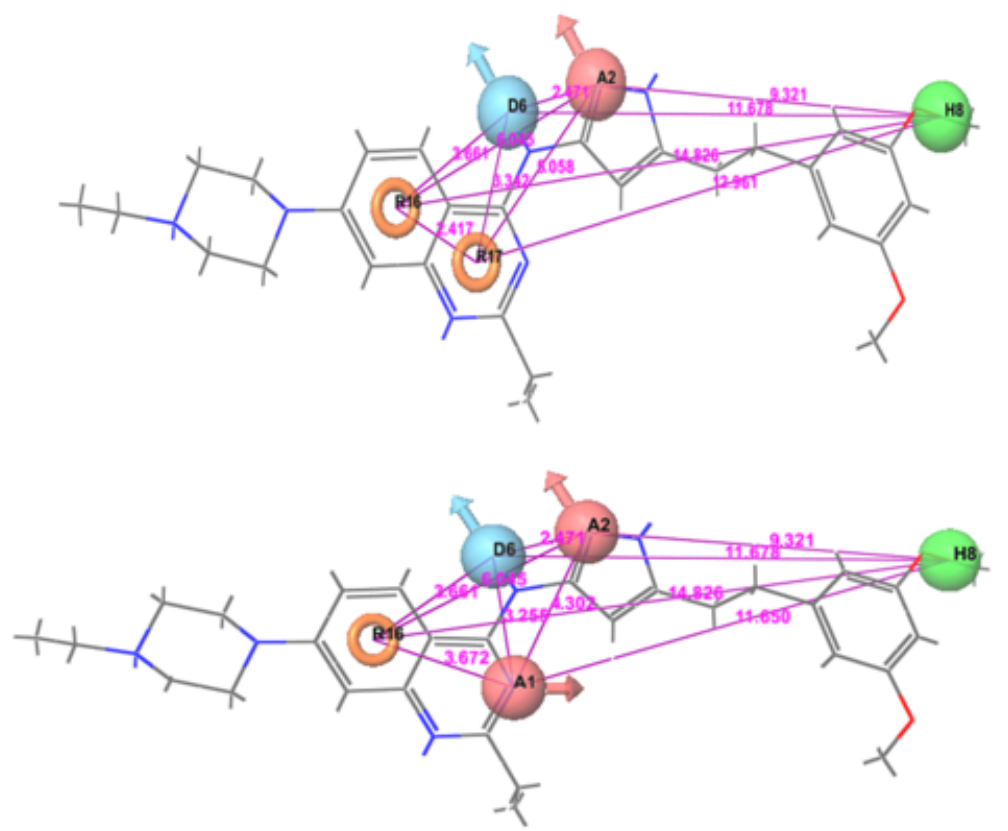

(a)

Fig. 1. The pharmacophore hypotheses (a) ADHRR.2051 (acceptor (A2, pink), donor (D6, blue), hydrophobic (H8, green) and ring (R16, R17, orange)); (b) AADHR.798 (acceptor (A1, A2, pink), donor (D6, blue), hydrophobic ( $\mathrm{H} 8$, green) and ring (R16, orange)) aligned to the compound 5 (best fitness score $=3$ ) 


\begin{tabular}{|c|c|c|c|c|c|c|c|c|c|}
\hline ID & Survival score & Survactive score & Site & Vector & Volume & Selectivity & Energy & Activity & Inactive \\
\hline ADHRR.2051 & 3.647 & 1.933 & 0.91 & 0.993 & 0.747 & 1.693 & 0.735 & 9.301 & 1.714 \\
\hline AADHR.798 & 3.623 & 1.923 & 0.89 & 0.995 & 0.742 & 1.592 & 0.735 & 9.301 & 1.7 \\
\hline AADRR.1836 & 3.652 & 1.893 & 0.93 & 0.977 & 0.745 & 1.606 & 0.735 & 9.301 & 1.759 \\
\hline AAADR.339 & 3.628 & 1.866 & 0.92 & 0.977 & 0.736 & 1.398 & 0.735 & 9.301 & 1.763 \\
\hline AADRR.1837 & 3.637 & 1.822 & 0.93 & 0.977 & 0.727 & 1.606 & 1.444 & 9.699 & 1.815 \\
\hline
\end{tabular}

Table 2

THE BEST FIVE MODELS DISPLAYING THE HIGHEST SURVIVAL SCORE AND MATCHING ALL ACTIVE COMPOUNDS
Survival score - a weighted sum of site, vector, volume, and a term for the number of matches; survival - inactive -the difference in survival score between actives and inactives; site score - accounts for the proximity of site points in the ligands contributing to the hypothesis, based on the RMS deviation; vector alignment score - record the vectorial fitness for acceptors, donors, and aromatic rings; volume - measures the overlapping volumes of the contributing structures; selectivity - accounts of the infrequency of the hypothesis, according to World Drug Index; energy - the energy difference $(\mathrm{kcal} / \mathrm{mol})$ between the reference structure and the lowest energy structure; activity - activity of the reference ligand; inactive - the survival score of inactives.

\section{Atom-based 3D QSAR}

Scatter plots of the experimental versus predicted activities of pyrazolylaminoquinazoline derivatives, obtained for ADHRR.2051 and AADHR.798 at 3 PLS factors show good correlative properties and low difference between experimental and predicted values, and are presented in figure 2.

On the basis of the maximal fitness score, the compound 5 (fitness score $=3$ ) was selected as reference compound to perform further studies. In order to investigate the correlation of the most important interactions with biological activity the hydrogen bond donor, hydrophobic, electron withdrawing groups and positive ionic fields were generated for compound $\mathbf{5}$. In figures 3-6 the most favorable features are depicted in blue, whereas the detrimental features are indicated in red. We can observe several favorable donor fields localized on NH group, pyrazole and piperazine rings and one unfavorable hydrogen bond donor area on pyrimidine ring substituent (fig. 3). Large favorable hydrophobic areas extended on the whole molecule accompanied by several unfavorable regions in the central part of the molecule and on $\mathrm{H} 8$ can be noticed (fig. 4). Electron withdrawing favorable zones covers piperazine, pyrazole and phenyl ring substituents, but $\mathrm{H} 8$ and pyrimidine are partially covered by unfavorable areas (fig. 5). A favorable positive ionic area is located on pyperazine ring, whereas an unfavorable zone is observed on pyrimidine ring (fig. 6). Hydrogen bond donor and electron withdrawing fields suggests two favorable areas on $\mathrm{NH}$ and pyridazole ring which are susceptible to interact with the receptor.

\section{Docking}

The compound $\mathbf{5}$ showing the highest affinity towards FGFR2 was chosen to validate by rigid receptor - flexible ligand docking the results obtained by pharmacophore modeling and 3D atom-based QSAR. The acceptor $=\mathrm{N}$ and donor $\mathrm{NH}_{2}$ groups interact effectively with FGFR-2 binding site by making two hydrogen bonds with Ala567 (PDB ID: 1OEC) at $2.787 \AA$ and at $3.194 \AA[33,34]$ (see fig.7), similar to compound 7 docked into FGFR1 [14]. This was also suggested by pharmacophore hypotheses ADHRR.2051 and AADHR.798 where $=\mathrm{N}$ - corresponds to acceptor site $\mathrm{A} 2$ and $\mathrm{NH}_{2}$ coincide with donor site $\mathrm{D6}$. Hydrogen bond donor and electron withdrawing fields indicated D6 and A2 as favorable areas. The spatial arrangement of acceptor and donor sites inside ADHRR.2051 and ADHRR.798 hypotheses is supported also by the interactions observed into co-crystal structure of

\begin{tabular}{|c|c|c|c|c|c|c|c|c|}
\hline ID & PLS $^{ \pm}$ & SD & R-squared & F & P & RMSE & Q-squared & Pearson-R \\
\hline \multirow{4}{*}{ ADHRR.2051 } & 1 & 0.288 & 0.827 & 81.100 & $7.02 \mathrm{E}-08$ & 0.196 & 0.671 & 0.828 \\
\cline { 2 - 9 } & 2 & 0.190 & 0.929 & 105.100 & $6.27 \mathrm{E}-10$ & 0.223 & 0.576 & 0.776 \\
\cline { 2 - 9 } & 3 & 0.100 & 0.982 & 266.600 & $3.13 \mathrm{E}-13$ & 0.204 & 0.645 & 0.805 \\
\hline \multirow{4}{*}{ AADHR.798 } & 1 & 0.282 & 0.835 & 85.800 & $4.70 \mathrm{E}-08$ & 0.199 & 0.662 & 0.816 \\
\cline { 2 - 9 } & 2 & 0.173 & 0.942 & 128.800 & $1.37 \mathrm{E}-10$ & 0.218 & 0.592 & 0.781 \\
\cline { 2 - 9 } & 3 & 0.098 & 0.982 & 276.800 & $2.37 \mathrm{E}-13$ & 0.196 & 0.671 & 0.820 \\
\hline
\end{tabular}

\# Number of PLS factors; SD - standard deviation of the regression; R-squared - the coefficient of determination; $F$ - the ratio of the model variance to the observed activity variance; $P$ - the significance level of variance ratio; RMSE - the root-mean-squared error for the test set predictions; Q-squared - analogous to R-squared based on the test set predictions; Pearson- $\mathrm{R}$ - the value for the correlation between the predicted and observed activity for the test set [16-18]

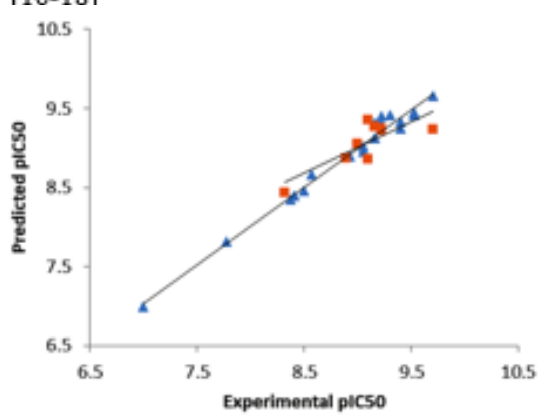

(a)

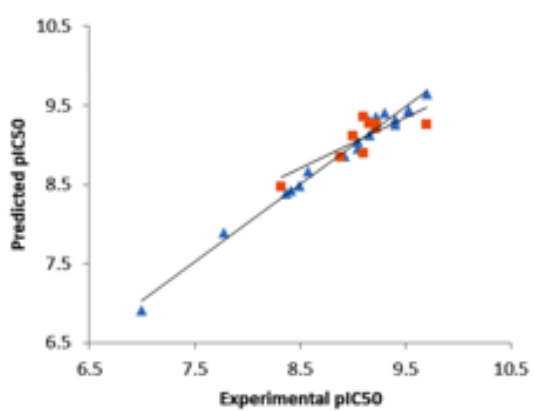

(b)
Table 3

THE STATISTICAL PARAMETERS OF THE ATOM-BASED 3D QSAR MODELS

Fig. 2. Experimental versus predicted $\begin{array}{ll}\text { A Training test } & \text { activities for 3D-QSAR } \\ \text { - Test set models (a) ADHRR2051 }\end{array}$ and (b) ADHRR798 


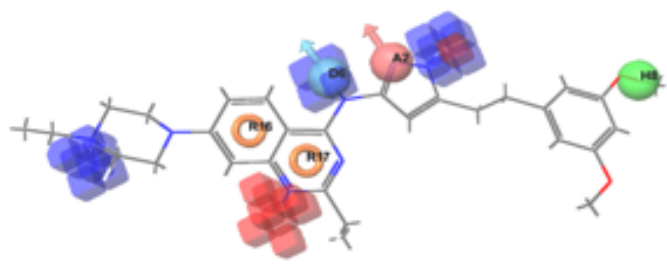

(a)

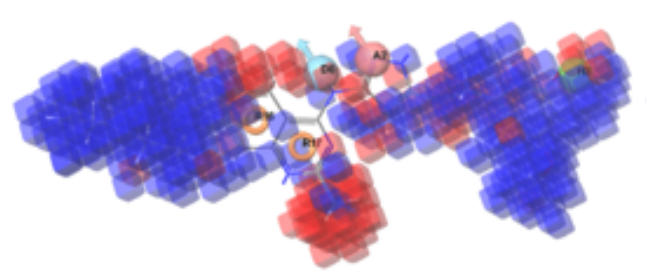

(a)

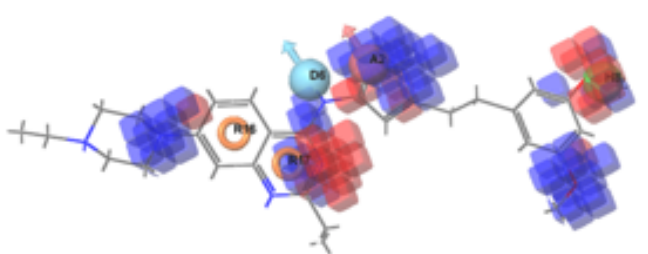

(a)

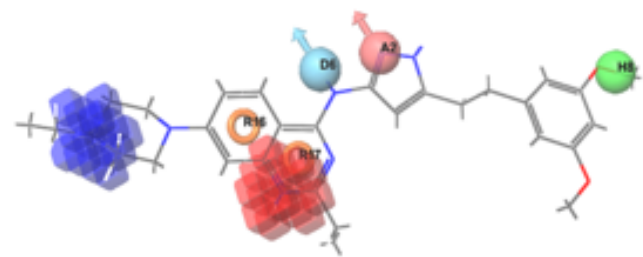

(a)

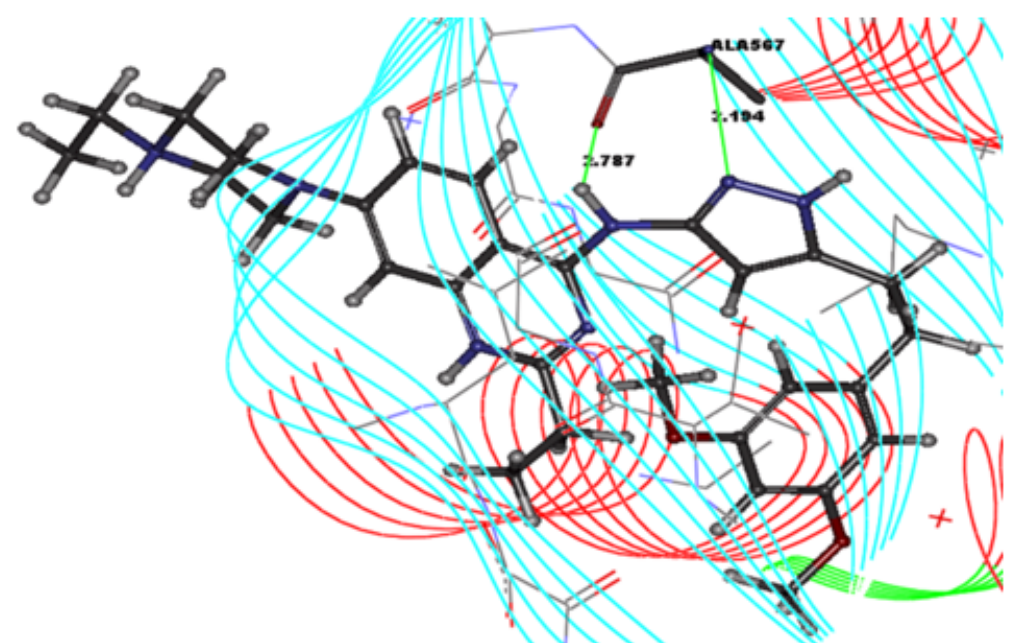

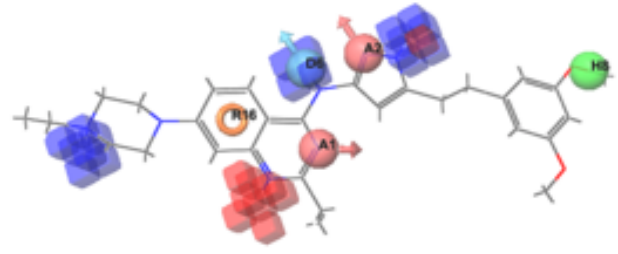

(b)
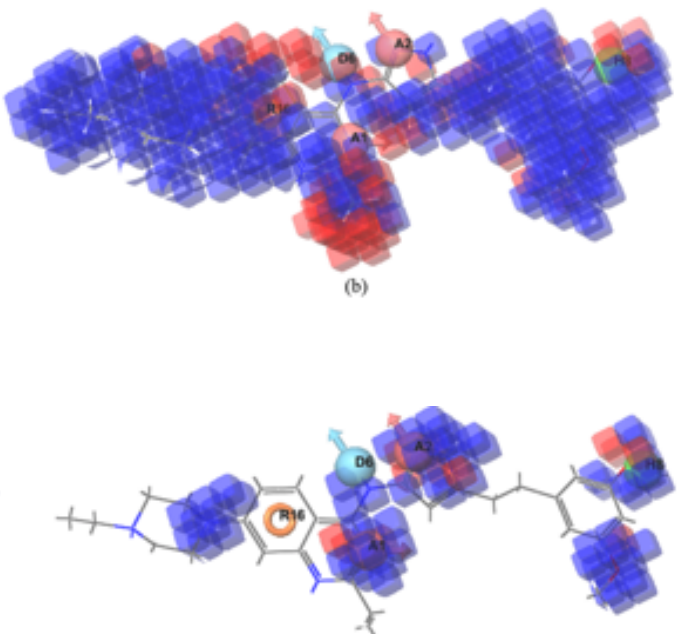

(b)

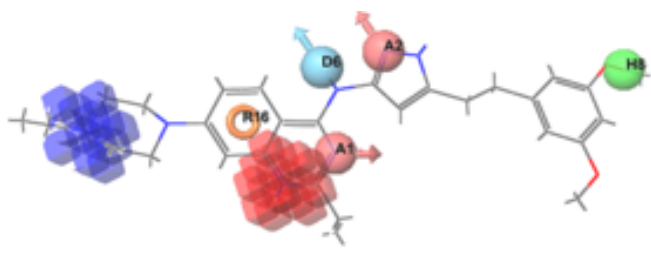

(b)
Fig. 3. Favorable (blue) and unfavorable (red) hydrogen bond donor fields for compound 5: (a) ADHRR.2051 and (b) ADHRR.798.
Fig. 4. Favorable (blue) and unfavorable (red) hydrophobic fields for compound 5: (a) ADHRR.2051 and (b) ADHRR.798.
Fig. 5. Favorable (blue) and unfavorable (red) electron withdrawing fields for compound 5: (a) ADHRR.2051 and (b) ADHRR.798.

Fig. 6. Favorable (blue) and unfavorable (red) positive ionic fields for compound $\mathbf{5}$ :

(a) ADHRR.2051 and (b) ADHRR.798.
FGFR2 with 4-aryl-2-phenylamino pyrimidine (PDBID: 10EC) [34] and (6S)-6-phenyl-5,6-dihydrobenzo[h] quinazolin- 2-amine (PDBID: 3RI1) [35] which form two hydrogen bonds with Ala567. Based on pharmacophore hypotheses, 3D atom-based QSAR and docking, pyrazolylaminoquinazoline inhibitors can interact similarly to the ligands in co-crystals of FGFR2 PDBID: 1OEC [34] and 3RI1 [35] which hamper the activation of the autoinhibited conformation of FGFR2 kinase. The ligands co-crystalized with FGFR2 in 10EC and 3RI1 co-crystals show significant inhibitory activity in cancer cells [35]. Since the inhibition of inactive kinases is an actual subject, the current investigation might provide a clue for the design of inhibitors that target the inactive conformations FGFR2 kinase.

\section{Conclusions}

In the current investigation, we used pharmacophore modeling, 3D atom-based QSAR and docking to investigate pyrazolylaminoquinazoline potent FGFR2 inhibitors. The computational approach was thought-out to provide insight into the structural features of pyrazolylaminoquinazoline derivatives which determine their biological activity against FGFR2. The selected pharmacophore hypotheses are in accord with experimental interactions observed in FGFR2 co-crystals with ligands (10EC and 3RI1), whereas their afferent atom-based 3D-QSAR models displayed good correlative and predictive abilities (R-squared $>0.9$, Qsquared $>0.6$, R-Pearson $>0.8$ ). Binding orientations and interaction features of these molecules with FGRF2 were also investigated by docking experiments. Indeed, the 
investigated compounds made contacts with critical residue Ala567, which was observed also in 10EC and 3RII co-crystals, showing a good agreement between docking results and experimental data. Our results can and can guide the design of novel pyrazolylaminoquinazoline analogs with improved properties.

Acknowledgements: The authors thank OpenEye Ltd. and Accelerys Software Inc. (for the Discovery Studio Visualiser v.2.5 program) for providing academic license and to Dr. Ramona Curpãn (Institute of Chemistry Timisoara of Romanian Academy), for providing access to Schrödinger software acquired through the PN-II-RU-TE-2014-4-422 projects funded by CNCS-UEFISCDI Romania. This project was financially supported by Project 1.2 of the Institute of Chemistry of the Romanian Academy.

\section{References}

1. ESWARAKUMAR, V.P., LAX, I., SCHLESSINGER, J., Cytokine Growth Factor Rev., 16, 2005, p. 139

2. PATEL, V.N., REBUSTINI, I.T., HOFFMAN, M.P., Differentiation, 74, 2006, p. 349

3. GUO, M., LIU, W., SERRA, S., ASA, S.L., EZZA, S., Cancer Res., 72, 2012, p. 2017

4. XU, R., RUDD, T.R., HUGHES, A.J ., SILIGARDI, G., FERNIG, D.G., YATES, E.A., FEBS J ournal, 280, 2013, p. 2260

5. BHADORIYA, K.S., SHARMA, M.C., SHARMA, S., JAIN, S.V., AVCHAR, M.H., Arab. J. Chem., 7, 2014, p. 924

6. J AIN, S.V., BHADORIYA, K.S., BARI, S.B., SAHU, N.K., GHATE, M., Med. Chem. Res., 21, 2012, p. 3465

7. JAIN, S.V., GHATE, M., BHADORIYA, K.S., BARI, S.B., CHAUDHARI, A., BORSE, J.S., Org. Med. Chem. Lett, 2, 2012, p. 22

8. SHARMA, M.C., SHARMA, S., SHARMA, P., KUMAR, A., BHADORIYA, K.S., J. Taiwan Inst. Chem. Eng., 45, 2014, p. 12

9. BHADORIYA, K.S., SHARMA, M.C., JAIN, S.V., J. Mol. Struct., 1081, 2015, p. 466

10. AVRAM, S., AVRAM, S., DEHELEAN, C., Rev. Chim. (Bucharest), 67, no.1, 2016, p. 119

11. JAIN, S.V., BHADORIYA, K.S., BARI, S.B., Med. Chem. Res., 2012,

21, 1665-1676.

12. KELLER, P.A., BIRCH, C., LEACH, S.P., TYSSEN, D., GRIFFITH, R., J . Mol. Graph. Model., 21, 2003, p. 365

13. IVAN, D., CRISAN, L., PACUREANU, L., Rev. Chim. (Bucharest), 62 , no.8, 2011, p. 806

14. JUN, F., YANG, D., JINGWEI, S., XIA, P., CHEN, W., SUFEN, C., BIN, Z., JING, A., MEIYU, G., WENHU, D., Bioorg. Med. Chem. Lett., 26, 2016, p. 2594
15. *** Symyx Draw version 3.3, http://symyx draw.software. informer.com/3.3/, 2010.

16. DIXON, S.L., SMONDYREV, A.M., KNOLL, E.H., RAO, S.N., SHAW, D.E., FRIESNER, R.A., J. Comput. Aided. Mol. Des., 20, 2006, p. 647 17. DIXON, S.L., SMONDYREV, A.M., RAO, S.N., Chem. Biol. Drug. Des., 67, 2006, 370

18. *** Schrödinger Release 2016-1: Phase, Schrodinger, LLC, New York, NY, 2016.

19.*** Small-Molecule Drug Discovery Suite 2016-1, Schrodinger, LLC, New York, NY, 2016.

20. *** Schrödinger Release 2016-1:LigPrep, Schrodinger, LLC, New York, NY, 2016.

21. WATTS, K.S., DALAL, P., MURPHY, R.B., SHERMAN, W., FRIESNER, R.A., SHELLEY, J.C., J. Chem. Inf. Model., 50, 2010, p. 534

22. *** Schrödinger Release 2016-1:ConfGen, Schrödinger, LLC, New York, NY, 2016.

23.*** OpenEye Scientific Software; Omega version 2.5.1.4, Santa Fe NM 87508; http://www.eyesopen.com, 2013.

24. HAW KINS, P.C.D., SKILLMAN, A.G., WARREN, G.L., ELLINGSON, B.A., STAHL, M.T., J. Chem. Inf. Model., 50, 2010, p. 572

25. HAW KINS, P.C.D., NICHOLLS, A.N., J. Chem. Inf. Model., 52,

2012, p. 2919

26. PEROLA, E., CHARIFSON, P.S., J. Med. Chem., 47, 2004, p. 2499

27. KRISTAM, R., GILLET, V.J., LEWIS, R.A., THORNER, D., J. Chem. Inf. Model., 45, 2005, p. 461

28. *** http://www.rcsb.org/pdb/explore/

explore.do?structureld=10EC, accessed in November 2016

29. OpenEye Scientific Software; Fred Receptor module version 2.2.5, Santa Fe NM 87508; http://www.eyesopen.com, 2013.

30. *** OpenEye Scientific Software; OEDocking version 3.0.1, Santa Fe NM 87508; http://www.eyesopen.com, 2012.

31. McGANN, M., J. Chem. Inf. Model., 51, 2011, p. 578

32. MCGANN, M.R., ALMOND, H.R., NICHOLLS, A., GRANT, J.A., BROWN, F.K., Biopolymers, 68, 2003, p. 76

33. *** Discovery Studio Visualizer-Accelrys version 2.5 San Diego, CA. http://accelrys.com, 2009.

34. T.A. CESKA, R. OWENS, C. DOYLE, P. HAMLYN, T. CRABBE, D. MOFFAT, J. DAVIS, R. MARTIN, M.J. PERRY, The Crystal Structure of the FGFR2 Tyrosine Kinase Domain in Complex with 4-aryl-2-

phenylamino pyrimidine Angiogenesis Inhibitors, To be published. 35. EATHIRAI , S., PALMA, R., HIRSCHI, M., VOLCKOVA, E., NAKUCI, E., CASTRO, J., CHEN, C.R., CHAN, T.C., FRANCE, D.S., ASHWELL, M.A., J.Biol.Chem., 286, 2011, p. 20677

Manuscript received: 23.05 .2018 
